\title{
Highly Effective Total Synthesis of Benzofuran Natural Product Egonol
}

\author{
Da Hye Choi, Jung Woon Hwang, Hyun Suck Lee, Deok Mo Yang, and Jong-Gab Jun
}

\author{
Department of Chemistry and Institute of Natural Medicine, Hallym Universin, Chuncheon 200-702. Korea \\ ${ }^{*}$ E-mail: jgimiähallwm.ack $\mathrm{n}$ \\ Received Mav $1+2008$
}

Key Words : Egonol. Benzofuran. Styracaceae. Sonogashira coupling. Iodovanillin

Benzo[b]furan natural products isolated from the Styracaceae family such as Strrax japonicum. ${ }^{3} S$ formosanis. ${ }^{2} S$. obassia. ${ }^{3}$ S. macranthis ${ }^{4}$ and S. officinalis show variety of biological activities including insecticidal. fungicidal, antimicrobial antisweet. antiproliferative. cytotoxic and antioxidant properties. Egonol. 5-(3-hy'droxypropyl)-7-methoxy-2-(3.4-methylenedioxyphenyl)benzofuran. was first isolated in 1915 from the seed oil of Strox japonicum ${ }^{l}$ and furst synthesized by $\mathrm{Kawai}^{7}$ condensing an $o$-hydroxybenzaldehyde with an $\alpha$-chlorophenylacetic acid and known to be an effective pyrethrum synergist. ${ }^{\delta}$ A number of total synthesis of egonol have been reported by using Sonogashira coupling . Lewis acid induced dehydrocyclization. dienylacetylene coupling with carbene complex. ${ }^{1]}$ and palladium-catalyzed cross-coupling reaction." We report herein the most effective total sy'nthesis of egonol (1. Figure 1) in 5 steps with $74 \%$ overall yield from vanillin by using Sonogashira coupling reaction.

Vanillin (2) reacted with $\mathrm{I}_{2} / \mathrm{Ag}_{2} \mathrm{SO}_{4}$ in $\mathrm{EtOH}$ at room temperature to give iodovanillin 3 in $80 \%$ yield (Scheme 1). Sonogashira coupling of 3 with 3.4-methylenedioxy phenylacetylene (4), which was easily prepared from piperonal via Colvin rearrangement. ${ }^{13}$ by using $\mathrm{Pd}\left(\mathrm{PPh}_{3}\right)_{4} / \mathrm{CuI} / \mathrm{Et}_{3} \mathrm{~N}$ in DMF y ielded benzofuran 5 in $95 \%$ yield through successive coupling and cyclization in one-step. Sonogashira coupling reactions were very sensitive to the haloaryl substituents as shown in Scheme 2. Bromovanillin 8 was not reactive with acetylene 4 in Sonogashira coupling reaction. and Wittig coupled bromide 9 and iodide 10 were also not reactive with 4 in coupling reaction. which indicated that the reaction sequences were very important in egonol synthesis and Sonogashira coupling should be done before Wittig reaction. Wittig reaction of $\mathbf{5}$ with (carbethoxymethy lene)triphenylphosphorane produced carbethoxyethenylbenzofuran 6 in $99 \%$ yield, which was reduced to 7 in $99 \%$ yield by $\mathrm{H}_{2} / \mathrm{Pd}-$ C. Direct reduction of conjugated ester 6 to egonol 1 was performed by using $\mathrm{LiAlH}_{4}$ or $\mathrm{LiBH}_{4}$ as shown in Scheme 3 . Partially reduced alcohol 11 with egonol and some other byproducts were formed in both cases and it was not easy to separate egonol from the alcohol 11 by column chromatography. Finally. reduction of ester 7 with DIBAL-H gave egonol (1) in $99 \%$ yield.

Khan isolated a new egonol derivative. 5-(3-propanoyloxypropyl)-7-methoxy-2-(3,4-methylenedioxy phenyl)benzofuran (12). from Stirax obassia (Scheme 4). ${ }^{14}$ The newly found benzofuran 12 was easily prepared from our synthetic egonol 1 by esterification with propanoic acid using DCC in $99 \%$ yield and confurmed by NMR data with literature.

In conclusion. the 5 steps reaction procedures including iodination. Sonogashira coupling. Wittig reaction, hỵdrogenation and metal hỵdride reduction produced egonol in

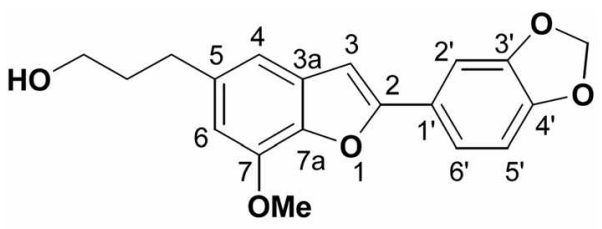

1

Figure 1. Chemical structure of egonol.

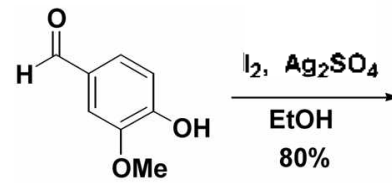<smiles>CCOC(=O)/C=C/c1cc(OC)c2oc(-c3ccc4c(c3)OCO4)cc2c1</smiles>

6

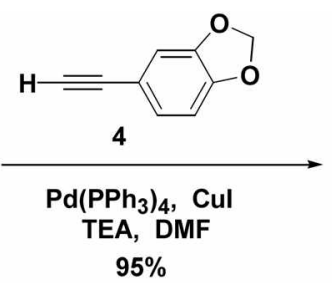

3

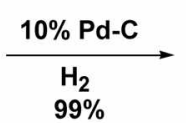

$99 \%$<smiles>COc1cc(C=O)cc2cc(-c3ccc4c(c3)OCO4)oc12</smiles>

5<smiles>CCOC(=O)CCc1cc(OC)c2oc(-c3ccc4c(c3)OCO4)cc2c1</smiles>

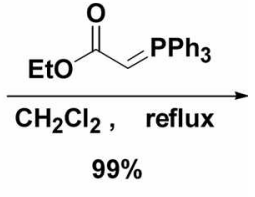

$99 \%$ 1 
<smiles>COc1cc(C=O)cc(Br)c1O</smiles><smiles>C#Cc1ccc2c(c1)OCO2</smiles><smiles>CCOC(=O)/C=C/c1cc(Br)c(O)c(OC)c1</smiles><smiles>C#Cc1ccc2c(c1)OCO2</smiles><smiles>CCOC(=O)/C=C/c1cc(I)c(O)c(OC)c1</smiles>

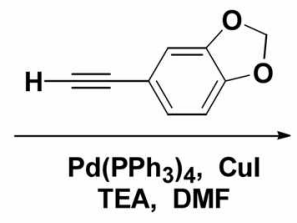

no reaction

10

Scheme 2

$74 \%$ overall yield from vanillin and the new benzofuran egonol derivative 12 was synthesized using this methodology for biological tests. This synthetic method provides the most effective egonol total synthesis in our best knowledge.

\section{Experimental Section}

All chenicals used were purchased from conmercial sources and used as received unless otherwise stated. NMR spectra were recorded at varian Mercury TM300 MHz FTNMR for ${ }^{1} \mathrm{H}$ and $75 \mathrm{MHz}$ for ${ }^{13} \mathrm{C}$, with the chemical shifts $(\delta)$ reported in parts per million (ppmi) relative to TMS and the coupling constants $(J)$ quoted in $\mathrm{Hz}$. $\mathrm{CDCl}_{3}$ was used as a solvent and an internal standard. Flash chromatography was carried out using silica gel Merck 60 (230-400 mesh). Thin-layer chromatography (TLC) was performed on DCPlastikfolien $60, F_{3-4}$ (Merck. layer thickness $0.2 \mathrm{~mm}$ ) plastic-backed silica gel plates with visualization by UV light (254 num) or by treatment with $p$-anisaldehyde. Melting points were measured on a MEL-TEMP II apparatus and were uncorrected.

4-Hydroxy-3-jodo-5-methoxybenzaldehyde (3). To a solution of vanillin (2) (l.00 g. $6.57 \mathrm{mmol})$ in EtOH $(50 \mathrm{~mL})$ under nitrogen atmosphere was added $\mathrm{I}_{2}(2.08 \mathrm{~g} .7 .89 \mathrm{mmol})$ with silver sulfate ( $2.46 \mathrm{~g} .7 .89 \mathrm{mmol}$ ) and stirred for $\mathrm{l}$ h at rt. Solvent was removed by evaporation and the organic product was extracted with $\mathrm{CH}_{2} \mathrm{Cl}_{2}$, washed with brine. dried and concentrated to give the solid. The solid was chromatographed (EtOAc:Hexane $=1: 2$ ) to give the white solid 3 $(1.47 \mathrm{~g} .80 \%), \mathrm{R}_{f} 0.34$ (EtOAc:Hexane = 1:3): mp 179-182 ${ }^{\circ} \mathrm{C},{ }^{1} \mathrm{H}$ NMR $\left(300 \mathrm{MHz}, \mathrm{CDCl}_{3}\right) \delta 3.97(3 \mathrm{H} . \mathrm{s}, \mathrm{OMe}) .6 .69$ (lH.s. OH). $7.36(\mathrm{lH}, \mathrm{d} . J=1.5 \mathrm{~Hz}, \mathrm{C} 6 \cdot \mathrm{H}) .7 .81(\mathrm{lH}, \mathrm{d} . J=$ $1.5 \mathrm{~Hz} . \mathrm{C} 2-\mathrm{H}), 9.75(\mathrm{lH}, \mathrm{s} . \mathrm{CHO}):{ }^{13} \mathrm{C}$ NMR $(75 \mathrm{MHz}$, $\left.\mathrm{CDCl}_{3}\right) \delta 56.8(\mathrm{OMe}), 80.7(\mathrm{C} 3-\mathrm{I}), 108.8(\mathrm{C} 6), 131.2(\mathrm{Cl})$, $136.4(\mathrm{C} 2), 146.6(\mathrm{C} 5), 151.5(\mathrm{C} 4), 189.7(\mathrm{C}=\mathrm{O})$.

5-Formyl-7-methoxy-2-(3,4-methylenedioxyphenyl)benzofuran (5). To a solution of iodovanillin (3) $(0.06 \mathrm{~g}$. $0.22 \mathrm{mmol}), \mathrm{Pd}\left(\mathrm{PPl}_{3}\right)_{4}(0.014 \mathrm{~g}, 0.02 \mathrm{mmol})$. 3,4-methylenedioxyphenylacetylene $(t, 0.05 \mathrm{~g} .0 .32 \mathrm{mmol})$ and $\mathrm{CuI}$ (0.001 g. $0.004 \mathrm{mmol})$ in DMF (3 $\mathrm{mL})$ under nitrogen atmosphere was added $\mathrm{Et}_{3} \mathrm{~N}(0.03 \mathrm{~mL} .0 .22 \mathrm{mmol})$ and stirred for $15 \mathrm{~h}$ at $\mathrm{rt}$. The organic product was extracted with $\mathrm{CHCl}_{3}$. washed with brine. dried and concentrated to give the solid. The solid was chromatographed (EtOAc:Hexane = 1:4) to give the yellow solid $5(0.04$ g. $95 \%) . R_{f} 0.28$ (EtOAc:Hexane = 1:3): mp 176-178 ${ }^{\circ} \mathrm{C}:{ }^{1} \mathrm{H}$ NMR $(300$ $\left.\mathrm{MHz}_{2} \mathrm{CDCl}_{3}\right) \delta 4.08(3 \mathrm{H} . \mathrm{s}, \mathrm{OMe}) .6 .02\left(2 \mathrm{H}, \mathrm{s}, \mathrm{O}-\mathrm{CH}_{2}-\mathrm{O}\right)$, 6.88 (lH. d. $J=8.4 \mathrm{~Hz}, \mathrm{C} 5^{\prime}-\mathrm{H}$ ). 6.93 (lH, s. C $\left.3-\mathrm{H}\right) .7 .31$ (lH. d. $\left.J=1.8 \mathrm{~Hz}, \mathrm{C} 22^{\prime}-\mathrm{H}\right) .7 .33(\mathrm{lH}$. br d. C6-H). $7.41(\mathrm{lH}$. dd, $\left.J=1.8 .8 .0 \mathrm{~Hz} . \mathrm{C}^{\prime}-\mathrm{H}\right) .7 .66(\mathrm{lH}, \mathrm{d} . J=0.6 \mathrm{~Hz} . \mathrm{C} 4-\mathrm{H})$, 9.97 (lH. s, CHO): ${ }^{12} \mathrm{C}$ NMR (75 MHz. CDCl 3$) \delta 56.5(\mathrm{OMe})$. $100.9(\mathrm{C} 3), 101.7\left(\mathrm{O}-\mathrm{CH}_{2}-\mathrm{O}\right), 104.6(\mathrm{C} 2), 105.8$ (C6), 108.2 (C5). 109.0 (C5'). 119.3 (C4). $119.8\left(\mathrm{C}^{\prime}\right), 123.9\left(\mathrm{Cl}^{\prime}\right)$, 131.1 (C3a), $133.6(\mathrm{C} 7 \mathrm{a}) .146 .1(\mathrm{C} 7), 148.4(\mathrm{C} 4), 148.8$ (C3). $157.8(\mathrm{C} 2), 191.9(\mathrm{CHO})$.

5-Carbethoxyethenyl-7-methoxy-2-(3,4-methylenedioxyphenyl)benzofuran (6). To a solution of $5(0.05 \mathrm{~g}, 0.17$ mumol) in $\mathrm{CH}_{2} \mathrm{Cl}_{2}(5 \mathrm{~mL})$ under nitrogen atmosphere was added (carbethoxymethylene)triphenylphosphorane $(0.09 \mathrm{~g}$. $0.25 \mathrm{mmol}$ ) and refluxed for $7 \mathrm{~h}$. The organic product was extracted with $\mathrm{CH}_{2} \mathrm{Cl}_{2}$. washed with brine, dried and con-<smiles>CCOC(=O)/C=C/c1cc(OC)c2oc(-c3ccc4c(c3)OCO4)cc2c1</smiles>

6<smiles>COc1cc(CCCO)cc2cc(-c3ccc4c(c3)OCO4)oc12</smiles>

1

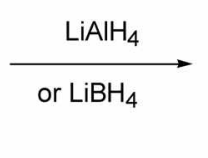

Scheme 3

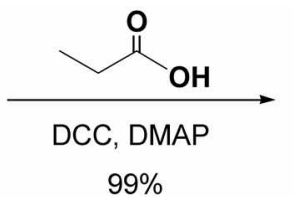<smiles>CCC(=O)OCCCc1cc(OC)c2oc(-c3ccc4c(c3)OCO4)cc2c1</smiles>

12 
centrated to give the solid. The solid was chromatographed (EtOAc:Hexane $=1: 3$ ) to give the white solid $6(0.062 \mathrm{~g}$. $99 \%) . R_{f} 0.44\left(\right.$ EtOAc:Hexane $=13$ ): $\mathrm{mp} 160-162{ }^{\circ} \mathrm{C} ;{ }^{1} \mathrm{H}$ NMR ( $\left.300 \mathrm{MHz}, \mathrm{CDCl}_{3}\right) \delta 1.35$ (3H. t. $\left.J=7.2 \mathrm{~Hz} . \mathrm{CH}_{3}\right)$. $4.05(3 \mathrm{H}, \mathrm{s} . \mathrm{OMe}), 4.27\left(2 \mathrm{H}, \mathrm{q} . J=7.2 \mathrm{~Hz} . \mathrm{OCH}_{2}\right), 6.01$ $\left(2 \mathrm{H}\right.$. s. $\left.\mathrm{O}-\mathrm{CH}_{z}=\mathrm{O}\right), 6.39(\mathrm{lH} . \mathrm{d} . J=15.6 \mathrm{~Hz}$, trans ethenyl Cl-H), 6.83 (lH. s. C6-H). 6.86 (lH. d. $J=8.4$ Hz. C $5^{\prime}-\mathrm{H}$ ). $6.94(1 \mathrm{H}$, br s, $\mathrm{C} 3-\mathrm{H}), 7.30\left(2 \mathrm{H}\right.$. br s. $\left.\mathrm{C} 22^{\prime} \cdot \mathrm{H} . \mathrm{C}+\mathrm{H}\right) .7 .39$ $\left(1 \mathrm{H}, \mathrm{dd} . J=1.5 .8 .7 \mathrm{~Hz} . \mathrm{C}^{\prime}-\mathrm{H}\right), 7.73(\mathrm{lH}, \mathrm{d}, J=15.9 \mathrm{~Hz}$. trans ethenyl $\mathrm{C} 2-\mathrm{H}):{ }^{13} \mathrm{C}$ NMR $\left(75 \mathrm{MHz} . \mathrm{CDCl}_{3}\right) \delta 14.8$ $\left(\mathrm{CH}_{3}\right) .56 .4\left(\mathrm{OCH}_{3}\right), 60.7\left(\mathrm{OCH}_{2}\right), 100.7(\mathrm{C} 3), 101.6(\mathrm{O}-$ $\left.\mathrm{CH}_{2}=\mathrm{O}\right) .105 .5\left(\mathrm{C}^{\prime}\right), 105.8(\mathrm{C} 6), 108.9\left(\mathrm{C}^{\prime}\right), 114.7\left(\mathrm{C}^{\prime}\right)$. 117.1 (ethenyl $\mathrm{Cl}$ ). 119.6 (C6'), $124.3\left(\mathrm{Cl}^{\prime}\right) .128 .8\left(\mathrm{C}^{\prime}\right)$. 130.8 (C3a). 131.5 (C7a). 145.3 (C7), 145.5 (ethenyl C2). $148.3\left(\mathrm{C}^{\prime}\right), 148.5\left(\mathrm{C}^{\prime}\right) .157 .1(\mathrm{C} 2), 167.3(\mathrm{C}=\mathrm{O})$.

5-Carbethoxyethyl-7-methoxy-2-(3,4-methylenedioxyphenyl)benzofuran (7). To a solution of $6(0.07 \mathrm{~g}, 1.19$ mmol) in $\mathrm{MeOH}(\mathrm{l} \mathrm{mL})$ under hydrogen balloon atmosphere was added Pd-C $(0.09 \mathrm{~g}, 10 \mathrm{wt} \%$ dry basis on activated carbon) and stirred for $3 \mathrm{~h}$ at $\mathrm{rt}$. The organic product was filterd and concentrated to give the solid. The solid was chromatographed (EtOAc:Hexane $=1: 3$ ) to give the white solid $7(0.07 \mathrm{~g} .99 \%) . R_{f} 0.60$ (EtOAc:Hexane $=$ 1:2): $\mathrm{mp} 88-90^{\circ} \mathrm{C}:{ }^{1} \mathrm{H}$ NMR $\left(300 \mathrm{MHz} . \mathrm{CDCl}_{3}\right) \delta 1.24(3 \mathrm{H}$. t. $\left.J=7.2 \mathrm{~Hz}_{2} \mathrm{CH}_{3}\right) .2 .66\left(2 \mathrm{H} . \mathrm{t}, J=8.1 \mathrm{~Hz} . \mathrm{CH}_{2}\right), 3.0 \mathrm{l}(2 \mathrm{H}$, t. $\left.J=8.1 \mathrm{~Hz} . \mathrm{CH}_{3}\right), 4.02\left(3 \mathrm{H}, \mathrm{s} . \mathrm{OCH}_{3}\right), 4.13(2 \mathrm{H}, \mathrm{q}, J=7.1$ $\left.\mathrm{Hz}, \mathrm{OCH}_{2}\right), 6.00\left(2 \mathrm{H}\right.$. s. $\left.\mathrm{O}-\mathrm{CH}_{2}-\mathrm{O}\right), 6.62(1 \mathrm{H}$. br s. $\mathrm{C} 6-\mathrm{H})$. $6.78(1 \mathrm{H}, \mathrm{s}, \mathrm{C} 3-\mathrm{H}), 6.85$ (1H. d. $\left.J=8.4 \mathrm{~Hz}, \mathrm{C} 55^{\prime}-\mathrm{H}\right) .6 .96$ $(1 \mathrm{H}$, br s, $\mathrm{C}+\mathrm{H}), 7.30\left(1 \mathrm{H}, \mathrm{d} . J=1.8 \mathrm{~Hz} . \mathrm{C}^{\prime} \cdot \mathrm{H}\right) .7 .38(1 \mathrm{H}$. dd. $\left.J=8.1,1.5 \mathrm{~Hz}, \mathrm{C}^{\prime}-\mathrm{H}\right):{ }^{13} \mathrm{C}$ NMR $\left(75 \mathrm{MHz}, \mathrm{CDCl}_{3}\right) \delta$ $14.0\left(\mathrm{CH}_{3}\right) .31 .1\left(\mathrm{CH}_{2}\right), 36.3\left(\mathrm{CH}_{2}\right), 55.8\left(\mathrm{OCH}_{3}\right), 60.1$ $\left(\mathrm{OCH}_{5}\right) .100 .0(\mathrm{C} 3) .100 .9\left(\mathrm{O}_{-} \mathrm{CH}_{2}=\mathrm{O}\right), 105.1\left(\mathrm{C}^{\prime}\right) .106 .8$ (C6). $108.2\left(\mathrm{C}^{\prime}\right), 111.8(\mathrm{C} 4), 118.8\left(\mathrm{C}^{\prime}\right) .124 .2\left(\mathrm{Cl}^{\prime}\right), 130.6$ (C3a), 135.9 (x2. C5. C7a), 144.3 (C7). 147.5 (C4'). 147.6 (C3'). $155.6(\mathrm{C} 2) .172 .5(\mathrm{C}=\mathrm{O})$.

Egonol (1). To a solution of $7(0.040 \mathrm{~g} .0 .11 \mathrm{mmol})$ in THF $(4 \mathrm{~mL})$ under nitrogen atmosphere was added DIBAL$\mathrm{H}(1.0 \mathrm{M}, 0.25 \mathrm{~mL})$ and stirred for $1.5 \mathrm{~h}$ at $\mathrm{rt}$. The reaction was quenched by addition of aqueous $\mathrm{Na}_{2} \mathrm{CO}_{3}$ solution. The organic product was extracted with $\mathrm{CH}_{2} \mathrm{Cl}_{3}$. washed with brine dried and concentrated to give the solid. The solid was chromatographed ( $\left.\mathrm{MeOH}: \mathrm{CHCl}_{\mathrm{3}}=1: 15\right)$ to give the yellow solid $1(0.035$ g. $99 \%)$. R $0.22($ EtOAc:Hexane $=1: 1) ; \mathrm{mp}$ $100-103^{\circ} \mathrm{C}:{ }^{1} \mathrm{H}$ NMR $\left(300 \mathrm{MHz}, \mathrm{CDCl}_{3}\right) \delta 1.94(2 \mathrm{H}$, quintet, $J=6.7 \mathrm{~Hz}$, propyl C2-H), 2.77 (2H. t. $J=7.5 \mathrm{~Hz}$, propyl ClH). $3.71\left(2 \mathrm{H}, \mathrm{t} . J=6.5 \mathrm{~Hz}, \mathrm{CH}_{2} \mathrm{OH}\right), 4.02\left(3 \mathrm{H}\right.$, s. $\left.\mathrm{OCH}_{3}\right)$. $5.99\left(2 \mathrm{H}, \mathrm{s}, \mathrm{O}-\mathrm{CH}_{2}-\mathrm{O}\right) .6 .62(1 \mathrm{H}$, br s. $\mathrm{C} 6-\mathrm{H}) .6 .77(\mathrm{lH} . \mathrm{s}$. $\mathrm{C} 3-\mathrm{H}), 6.85\left(1 \mathrm{H}, \mathrm{d}, J=7.8 \mathrm{~Hz}, \mathrm{C} 5{ }^{\prime}-\mathrm{H}\right), 6.95(1 \mathrm{H}$, br s, $\mathrm{C} 4$ H). $7.31\left(1 \mathrm{H}, \mathrm{d}, J=1.5 \mathrm{~Hz}, \mathrm{C} 2{ }^{\prime}-\mathrm{H}\right) .7 .38(1 \mathrm{H}, \mathrm{dd}, J=8.2 .1 .5$ Hz, C6'-H): ${ }^{13} \mathrm{C}$ NMR (75 MHz, $\mathrm{CDCl}_{3}$ ) $\delta 32.8$ (propyl C2). 35.1 (propyl Cl), $56.5\left(\mathrm{OCH}_{3}\right), 62.6\left(\mathrm{CH}_{3} \mathrm{OH}\right), 100.6(\mathrm{C} 3)$. $101.4\left(\mathrm{O}-\mathrm{CH}_{2}-\mathrm{O}\right) .101 .5\left(\mathrm{C}^{2}\right) .107 .6(\mathrm{C} 6), 108.8\left(\mathrm{C}^{\prime}\right)$. 112.5 (C4), $119.4\left(\mathrm{C}^{\prime}\right) .124 .9\left(\mathrm{Cl}^{\prime}\right) .131 .2(\mathrm{C} 3 \mathrm{a}) .137 .7$
(C5), $142.6(\mathrm{C} 7 \mathrm{a}), 144.9(\mathrm{C} 7) .148 .1\left(\mathrm{C}^{\prime}\right) .148 .2\left(\mathrm{C}^{\prime}\right)$. $156.2(\mathrm{C} 2)$.

5-(3-Propanoyloxypropyl)-7-methoxy-2-(3,4-methylenedioxyphenyl)benzofuran (12). To a solution of $1(0.010 \mathrm{~g}$. $0.031 \mathrm{mmol})$ in $\mathrm{CH}_{2} \mathrm{Cl}_{2}(5 \mathrm{~mL})$ under nitrogen atmosphere was added propionic acid $(0.045 \mathrm{~g} .0 .061 \mathrm{mmol}), N, N$ dicyclohexylcarbodiimide $(0.015 \mathrm{~g}, 0.077 \mathrm{mmol})$. dimethylaminopyridine $(0.00 \mathrm{~g}$ g. $0.0077 \mathrm{mmol})$ and refluxed for 10 $h$. The reaction mixture was filtered using celite filter and the organic product was extracted with $\mathrm{CH}_{2} \mathrm{Cl}_{2}$. washed with brine. dried and concentrated to give the solid. The solid was chromatographed (EtOAc:Hexane = 1:3) to give the white solid $12\left(0.01 \mathrm{l}\right.$ g. $99 \%$ ). $\mathrm{R}_{f} 0.63$ (EtOAc:Hexane = 1:2): $\mathrm{mp}$ $78-81^{\circ} \mathrm{C},{ }^{1} \mathrm{H}$ NMR $\left(300 \mathrm{MHz} . \mathrm{CDCl}_{3}\right) \delta 1.16$ (3H. t. $J=7.8$ Hz. Me). $1.99(2 \mathrm{H}$. quintet. $J=6.6 \mathrm{~Hz}$. propyl C $2-\mathrm{H}), 2.34$ $\left(2 \mathrm{H} . \mathrm{q}, J=7.5 \mathrm{~Hz} . \mathrm{CH}_{2} \mathrm{Me}\right), 2.74(2 \mathrm{H} . \mathrm{t}, J=7.5 \mathrm{~Hz}$, propyl $\mathrm{Cl}-\mathrm{H}), 4.02\left(3 \mathrm{H}, \mathrm{s}, \mathrm{OCH}_{3}\right), 4.12(2 \mathrm{H}, \mathrm{t} . J=6.6 \mathrm{~Hz}$, propyl C $3-\mathrm{H}) .5 .99\left(2 \mathrm{H} . \mathrm{s}, \mathrm{O}-\mathrm{CH}_{2}=\mathrm{O}\right) .6 .59(\mathrm{lH}, \mathrm{d}, J=1.2 \mathrm{~Hz}, \mathrm{C} 6-$ H), 6.77 (lH. s, C3-H), 6.85 (lH, d. $\left.J=7.8 \mathrm{~Hz} . \mathrm{C}^{\prime} \cdot \mathrm{H}\right) .6 .94$ (1H. br s. C4-H). $7.30(1 \mathrm{H}$. d. $J=1.5 \mathrm{~Hz}, \mathrm{C} 2 \mathrm{-H}) .7 .38(\mathrm{lH}$, dd $J=8.1 .1 .5 \mathrm{~Hz} . \mathrm{C}\left(6^{\prime}-\mathrm{H}\right){ }^{13} \mathrm{C}$ NMR $\left(75 \mathrm{MHz} . \mathrm{CDCl}_{3}\right) \delta$ $9.6(\mathrm{Me}), 28.0$ (propyl C2), 31.1 (propyl Cl). $32.9\left(\mathrm{COCH}_{2}\right)$, $56.5\left(\mathrm{OCH}_{3}\right) .64 .0$ (propyl C3). $100.6(\mathrm{C} 3), 101.5\left(\mathrm{O}-\mathrm{CH}_{2-}\right.$ O), $105.8\left(\mathrm{C}^{\prime}\right) .107 .6(\mathrm{C} 6) .108 .8\left(\mathrm{C}^{\prime}\right), 112.5(\mathrm{C} 4), 119.4$ (C6). 124.9 (Cl). 131.2 (C.3a). 137.1 (C.5). 142.7 (C.7a). 144.9 (C7), 148.1 (C4), $148.2\left(\mathrm{C}^{\prime}\right) .156 .3(\mathrm{C} 2) .174 .7(\mathrm{C}=\mathrm{O})$.

Acknowledgments. This work was supported by the Korea Research Foundation Grant funded by the Korean Government (MOEHRD. Basic Research Promotion Fund) (KRF-2007-412-J00501).

\section{References and Notes}

1. Okada. H. J. Pharn. Soc. Japan 1915.657.

2. Hopkins. C. Y.: Ewing. D. F.: Chisholm, M. J. Canad J. Chem. 1967. 45,1425

3. Takanashi. M: Takizawa. Y; Mitsuhashi. T. Chem. Lett 1974, 869

4. Luo. Y.: He. Z.: Li. H. Fitorerapia 2007. 78. 211

5. Segal. R.: Milo-Goldzweig. I.: Soholoff.' S.: Zitscek. D. V. J. Chent. Soc. (C) 1967.2402 .

6. Ward, R. S. Nat Prod Rep. 1999. 16.75.

7. Kawai. S.: Nakamura, T.: Sugiyama, N. Ber dt. Chem. Ges. 1939. 72. 1146.

8. Takanashi. M.: Takizawa. Y. Phytochenistry 1988. 27. 1224.

9. (a) Schreiber. F. G.: Stevenson. R. J. Cheni. Soc. Perkin Trans. $]$ 1976. 1514. (b) Wang. I.-I.: Lee. K.-I.: Kiml. M.-H.: Shim. Y. K. Bull. Korean Chem. Soc. 2004, 25, 1449

10. Choi, H.-D; Ha. M.-C.: Seo. P.-J.: Son. B.-W: Song, J.-C. Arch. Pham Res. 2000, 23. 438.

11. Zhang. J.: Zhang. Y.: Zhang. Y.: Herndon. J. W. Tetrahedron 2003. 59.5609 .

12. Aoyagi. Y: Mizusaki. T.: Hatori. A.: Asakura. T:: Aihara. T.: Inaba, S.: Hayatsu. K.: Ohta. A. Heterocycles 1995. H1. 1077.

13. Colvin. E. W.: Hamill. B. J. J. Chem. Soc. Perkin Trans. 1 1977. 869

14. Park. S. Y.: Lee. H.-J.: Lee. O.-K: Kang. H.-Y.: Choi. D.-H.; Paik. K.-H.: Khan. M. Bull. Konean Chent. Soc. 2007. 28. 1874. 